\title{
PROFESIONALIZACIÓN Y SENSIBILIZACIÓN DE LAS FUERZAS DE SEGURIDAD DEL ESTADO ANTE LA VIOLENCIA DE GÉNERO
}

\author{
Antonio Jesús Yugueros García \\ Doctor en Desarrollo y Ciudadanía \\ antyugue@botmail.com
}

1. Resulta de vital importancia conocer la opinión de los profesionales de la seguridad en relación con la violencia contra las mujeres en las relaciones de pareja o expareja.

Para los profesionales que atienden casos de violencia de género la asistencia a la víctima es lo primordial, lo más importante, ya que están ante una persona muy vulnerable que posiblemente lleve años sufriendo malos tratos de todo tipo y necesita una atención empática, actitud de escucha y de acogimiento, así como atender prontamente, si fuera necesario, tanto a su seguridad personal, como a la de sus hijos e hijas, y personas vulnerables que residan con ella.

La Ley Orgánica 1/2004, de 28 de diciembre, de Medidas de Protección Integral contra la Violencia de Género (LO 1/2004), ha sido un punto de inflexión en el tratamiento de este problema; ha constituido un antes y un después en la atención a las mujeres víctimas, ya que dispone la implementación, con carácter integral y multidisciplinar, de medidas encaminadas a que puedan salir de la situación en la que se encuentran y adquirir el empoderamiento perdido tras años de malos tratos.

Igualmente, han sido muy importantes los planes de sensibilización dirigidos a la población y a los profesionales, cada vez mejor cualificados y concienciados en este campo.

En este estudio se va a valorar la profesionalidad y sensibilización de los componentes de dos unidades de la Guardia Civil de la Comunidad Autónoma de Andalucía, que cotidianamente intervienen con mujeres víctimas de esta lacra social.

2. En primer lugar es conveniente apostillar, para entender los resultados del estudio, que el modelo policial español es mixto, es decir, que 
además de realizar las labores policiales propias de la prevención y represión del delito, las unidades policiales practican actividades, incluso más numerosas que las primeras enunciadas, de auxilios, rescates y otros servicios humanitarios.

Seguidamente se da cuenta del proceso metodológico seguido, así como del diseño de la estrategia de investigación que se ha considerado responde mejor a los objetivos propuestos y con ello aprobar o refutar la hipótesis de partida.

En relación con las muestras, se ha justificado su selección, los instrumentos y procesos de recogida de los datos, y la estrategia de análisis diseñada para la interpretación de los resultados.

En muchas ocasiones, las mujeres víctimas de estos execrables sucesos se personan en los cuarteles del cuerpo de la Guardia Civil, enclavados en las poblaciones donde residen, por ser el único sitio donde pueden acudir en caso de urgencia y recibir un auxilio inmediato. Otras veces acudirán con el objeto de solicitar información sobre sus derechos o cualquier otro aspecto derivado de la problemática que están padeciendo.

Ante esta realidad es necesario conocer la opinión de los guardias civiles sobre el servicio que prestan en este ámbito, y también otros aspectos y opiniones que les afectan. De esta forma se ha planteado la siguiente hipótesis:

Hipótesis. La Guardia Civil presta un servicio de calidad en el ámbito de la violencia de género en las relaciones de pareja o expareja. Esta posibilidad está basada en los criterios de mejor valoración expresados por la ciudadanía española y puestos de manifiesto en los barómetros del Centro de Investigaciones Sociológicas correspondientes a los meses de febrero y mayo de 2011 y abril de 2013 [CIS, 2011 a) y b); 2013].

Esta labor de asistencia y protección a las mujeres víctimas es posible que se desempeñe de mejor manera en los denominados puestos principales de la Guardia Civil, con especialistas específicos en violencia de género, en contraposición a los puestos ordinarios del cuerpo, que carecen de ellos, por poseer los primeros más medios y personal cualificado.

Objetivo general. Analizar la valoración de los agentes de la Guardia Civil que intervienen en este proceso de relación de asistencia; por ello, el objetivo específico de estudio es:

Objetivo específico 1. Analizar el grado de sensibilidad y profesionalidad de los guardias civiles en el ámbito de la violencia de género en las relaciones de pareja o expareja. 
Para responder a los objetivos de la investigación y aprobar o rectificar la hipótesis inicial se ha efectuado un planteamiento metodológico de esta investigación teniendo en cuenta lo enunciado por Rodríguez y Valldeoriolade (2009) cuando manifiestan que el pluralismo metodológico proporciona una diversidad metodológica que permite ampliar y perfeccionar la actividad del investigador. La decisión de optar por una u otra metodología de investigación vendrá determinada por el problema a investigar y por el método.

En esta investigación se ha optado por la articulación de métodos tanto cuantitativos como cualitativos, utilizando la triangulación metodológica, que consiste en la combinación de dos o más métodos de investigación que se complementan, con el fin de suplir las limitaciones y de la implementación de un solo método. Aunque en este artículo sólo se da cuenta del resultado del estudio cuantitativo.

El diseño de este estudio es de carácter transversal, porque se pregunta por unos temas concretos en un determinado momento.

Se trata de un estudio no experimental, descriptivo, porque no investiga las relaciones causa y efecto propias de las investigaciones empíricas, sino que en este caso lo que se hace es describir y analizar la relación entre variables.

La demarcación policial de actuación de la Guardia Civil es el ámbito rural. Esta investigación se ha llevado a cabo en dos municipios pertenecientes a la Comunidad Autónoma de Andalucía denominados «A $\mathrm{A} \ll \mathrm{B} »$. El primer municipio «A» posee más de 25.000 habitantes, teniendo enclavado en la localidad un puesto principal de la Guardia Civil con efectivos especialistas específicos en violencia de género en las relaciones de pareja o expareja, y el segundo municipio «B», con un número inferior a 25.000 habitantes, posee un puesto de la Guardia Civil ordinario.

A estos dos municipios se les ha denominado «A»y «B», respectivamente, para salvaguardar la identidad de todas las personas que han colaborado en el estudio.

El puesto de la Guardia Civil constituye la unidad territorial básica de seguridad ciudadana, la más próxima a la ciudadanía, garantizando de este modo una atención permanente y directa.

Atendiendo a diversas características de la demarcación, los puestos de la Guardia Civil se clasifican en principales y ordinarios. La concepción del puesto principal responde a la necesidad de dar una respuesta unificada y adecuada a la conflictividad de las localidades con predominio urbano, industrial o turístico situadas en la demarcación del cuerpo de la Guar- 
dia Civil, o bien en zonas de entidad y problemática homogénea que lo requieran por sus características demográficas, socioeconómicas, de seguridad ciudadana o similares. Poseen diferentes áreas para atención al ciudadano (DGGC, 2013), entre ellas las de violencia de género. Suelen estar establecidos en localidades con número de habitantes igual o superior a $\operatorname{los} 25.000$.

El puesto ordinario constituye la menor unidad de la Guardia Civil con capacidad para llevar a cabo las misiones encomendadas al cuerpo por la legislación vigente, en una demarcación de pequeña o mediana entidad de población, normalmente menor de 25.000 habitantes.

En este trabajo al puesto principal del municipio «A» se le denomina Unidad Policial A (UPA) y al puesto del municipio «B», Unidad Policial B (UPB).

Se quiere hacer constar que en esta investigación se alude habitualmente al masculino genérico cuando se hace referencia a los agentes de la Guardia Civil, porque casualmente no había ninguna mujer agente en estos destinos cuando se realizó este estudio.

Para la recogida de los datos cuantitativos se ha recurrido a cuestionarios estandarizados de preguntas, en su gran mayoría cerradas, administrados a los guardias civiles; de esta manera se ha permitido realizar una valoración más amplia del grado de sensibilización, profesionalidad y carencias de medios de los guardias civiles.

Se optó por realizar encuestas mediante cuestionario, porque permite obtener más opiniones de los guardias civiles, al poder acaparar una muestra de personas más amplia que con otros instrumentos de índole cualitativo como es la entrevista. Los cuestionarios han pasado por varias modificaciones desde su primera elaboración, habiéndose comprobado antes de ser utilizados.

Se han administrado 87 cuestionarios, estructurados con preguntas cerradas, a los guardias civiles de las localidades «A»y «B», que fueron facilitados a los agentes en sus unidades de destino respectivas, siendo informados previamente del objeto que se pretendía. Es conveniente anotar que las encuestas realizadas a los guardias civiles lo han sido a nivel personal, de forma voluntaria, sin que haya intervenido en el proceso la institución en forma alguna.

El cuestionario administrado a los guardias civiles pretende conseguir saber el grado de sensibilidad que tienen con respecto a la violencia de género en las relaciones de pareja o expareja y la opinión sobre la LO 1/2004 de Medidas de Protección Integral contra la Violencia de Género, entre otras consideraciones. 
Muestras

Poblaciones: en la presente investigación se han estudiado dos poblaciones:

1. Agentes de la Guardia Civil destinados en el municipio «A».

2. Agentes de la Guardia Civil destinados en el municipio «B».

Selección de las muestras

Los criterios que se tuvieron en cuenta para la selección de las muestras fueron los siguientes:

En cuanto a los guardias civiles:

- Ser agente en activo del cuerpo de la Guardia Civil.

- Estar destinado en los municipios objeto de estudio «A»y «B» en enero de 2013.

En relación con las poblaciones de los guardia civiles, en enero de 2013, en la localidad «A», había destinados 78 agentes (100 por 100) y en la localidad «B», 9 agentes (100 por 100).

Este estudio está sustentado en poblaciones pequeñas, porque la Guardia Civil actúa en poblaciones eminentemente de poca población.

Esta investigación, a pesar de que las muestras son pequeñas, ya que las poblaciones de estudio así lo son, se puede extrapolar al resto de las localidades de España con similar número de habitantes a los estudiados. Porque hay una correspondencia entre número de habitantes y casos de violencia de género visibilizados.

Estudio del grado de sensibilidad y conocimientos de los guardias civiles

Concepto de violencia de género. A los agentes se les ha preguntado sobre el concepto que tienen sobre la violencia de género, habiendo podido contestar:

- Es una lacra social que vulnera los derechos humanos de las mujeres.

- Es un invento para que las mujeres obtengan poder.

- Es exagerado, siempre ha ocurrido.

Las preguntas se han diseñado siendo extremadamente antagónicas entre sí, no dejando espacio a opiniones intermedias, que desde luego sur- 
gen. Esto ha sido así para facilitar que afloraran más fácilmente las contestaciones o bien estereotipadas, instauradas en el ideario social, o, por el contrario, libres de prejuicios y sensibilización ante esta problemática, fruto de la formación y la experiencia profesional que han ido adquiriendo los guardias civiles.

«Lacra social»

Unidades policiales agregadas:

- El 84 por 100 ( $n=73$ ) opinan que la violencia de género representa una lacra social.

- El 16 por 100 (n=14) se pronunció como que no lo era.

El gráfico 1 pone de manifiesto que en la UPA:

- El 82 por 100 ( $\mathrm{n}=64$ ) ha contestado que la violencia de género es una lacra social.

- El 18 por $100(n=14)$ no lo cree así.

\section{GRAFICO 1}

Distribución de la muestra de guardias civiles con los que opinan que la violencia de género es una lacra social. UPA

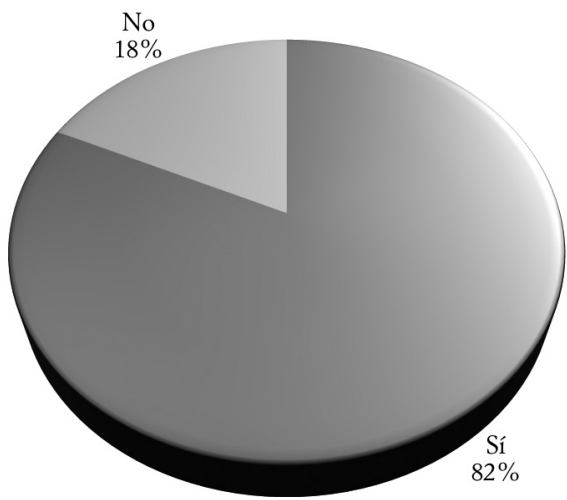

Fuente: elaboración propia, 2013.

En la unidad policial «B» el total de la plantilla [100 por 100 (n=9)] sí consideran la violencia de género como una lacra social. 
«Invento»

Unidades policiales agregadas:

- El 94 por $100(n=82)$ opina que no es un rollo ni invención para que las mujeres obtengan más poder.

- El 6 por $100(n=5)$ manifestó que sí lo es.

El gráfico 2 señala que en la UPA:

- El 94 por $100(n=73)$ de los guardias civiles ha manifestado que no era un invento,

- El 6 por $100(n=5)$ dijo que sí lo era.

En la UPB, el 100 por $100(n=9)$ de los efectivos dijo que la violencia de género no es un invento.

\section{«Exagerado»}

Unidades policiales agregadas:

- El 87 por $100(n=76)$ dice que no es exagerado.

- El 13 por $100(n=11)$ que opinan que sí lo es.

\section{GRAFICO 2}

Distribución de la muestra de los guardias civiles con los que opinan que la violencia de género es un invento. UPA

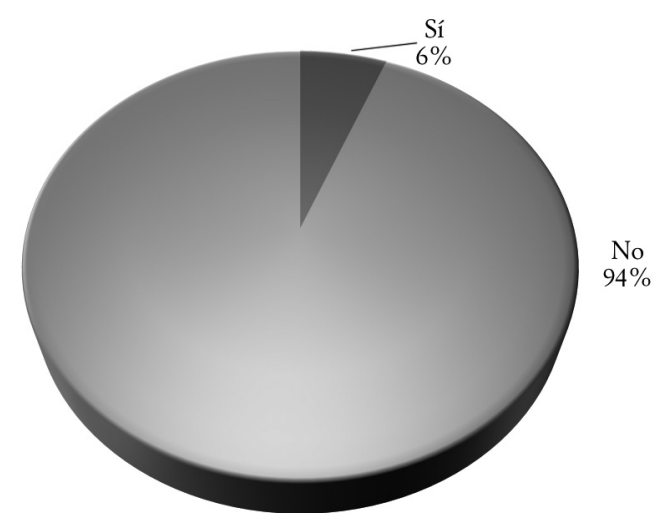

Fuente: elaboración propia, 2013. 


\section{GrÁFICO 3}

Distribución de la muestra de los guardias civiles con los que opinan que la violencia de género no es exagerada, siempre ba existido. UPA

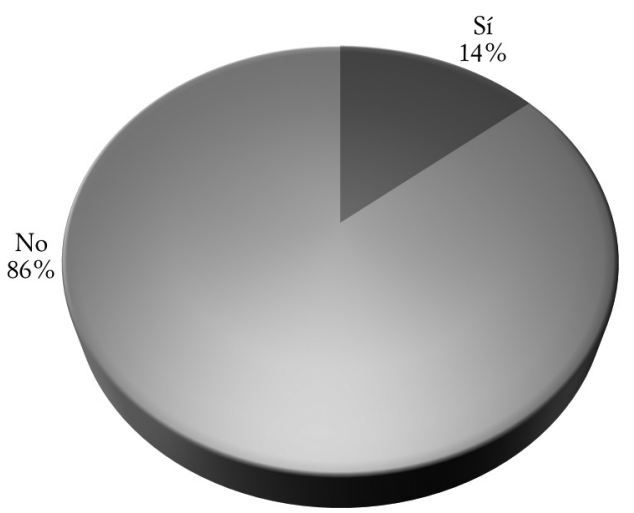

Fuente: elaboración propia, 2013.

El gráfico 3 expone que en la UPA:

- El 86 por 100 ( $\mathrm{n}=67$ ) ha manifestado que no es exagerado,

- El 14 por 100 ( $\mathrm{n}=11$ ) opina que sí lo es.

En la UPB, los resultados han sido del 100 por 100 (n=9) de sus efectivos en afirmar que no es exagerado.

Ley $1 / 2004$. En una segunda pregunta en esta misma línea se interesaba la opinión que a los agentes les merecía la LO 1/2004 de Medidas de Protección Integral contra la Violencia de Género, teniendo como posibles respuestas:

- Es una ley que va en contra de los hombres.

- Es utilizada por las mujeres para obtener beneficios de todo tipo.

- Ha sido de mucha utilidad para el desempeño de nuestro trabajo, ya que podemos prestar un mejor servicio a las mujeres víctimas.

De igual manera que en el caso anterior, las preguntas están basadas en los estereotipos que predominan en el diario social colectivo.

«Contra los hombres»

Unidades policiales agregadas:

- El 87 por 100 ( $\mathrm{n}=76)$ de los guardias civiles han manifestado que no va en contra de los hombres.

- El 13 por 100 (n=11) dijo que sí. 


\section{GRÁFICO 4}

Distribución de la muestra de los guardias civiles con los que opinan que la Ley 1/2004 va en contra de los hombres. UPA

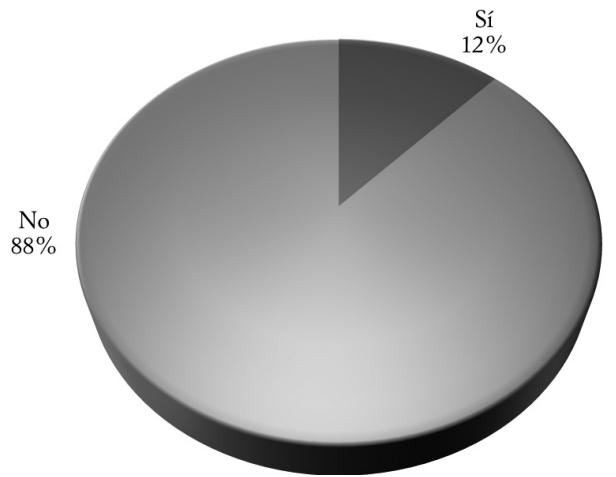

Fuente: elaboración propia, 2013.

El gráfico 4 indica que en la UPA:

- El 88 por $100(n=69)$ ha declarado que no es una ley que vaya en contra de los hombres, sino que es de mayor protección hacia las mujeres víctimas.

- El 12 por $100(n=9)$ piensa que sí es una ley restrictiva de derechos para los hombres.

\section{GRÁFICO 5}

Distribución de la muestra de los guardias civiles con los que opinan que la Ley 1/2004 va en contra de los hombres. UPB

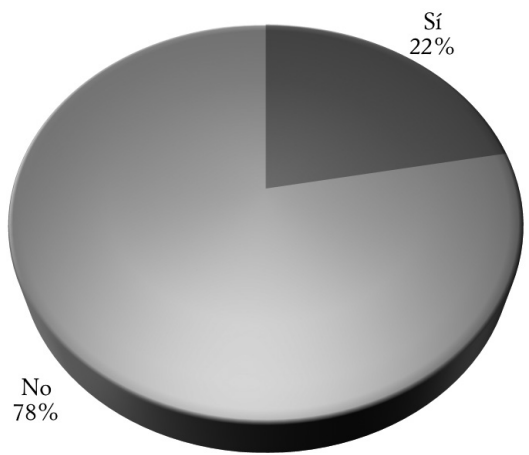

Fuente: elaboración propia, 2013. 
El gráfico 5 señala que en la UPB:

- El 78 por $100(n=7)$ opina que no es una ley en contra de los hombres.

- El 22 por 100 (n=2) dijo que sí lo era.

«Obtención de beneficios por parte de las mujeres»

Unidades policiales agregadas:

- El 80 por 100 ( $\mathrm{n}=70)$ contesta que no es utilizada por las mujeres solamente para obtener beneficios.

- El 20 por 100 (n=17) manifestó afirmativamente.

\section{GRÁFICO 6}

Distribución de la muestra de los guardias civiles con los que opinan que la Ley 1/2004 es utilizada por las mujeres para obtener beneficios, UPA

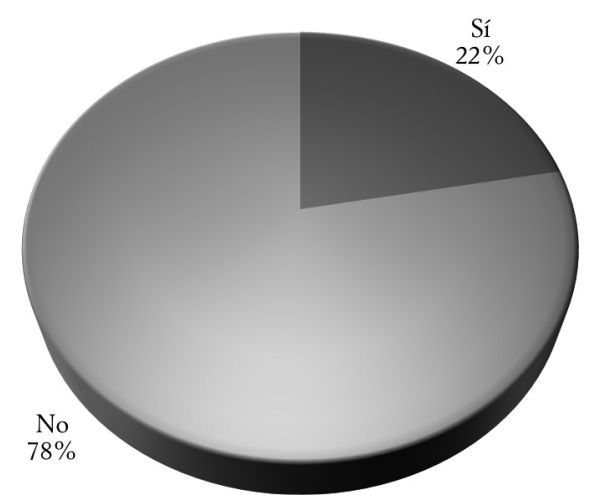

Fuente: elaboración propia, 2013.

El gráfico 6 señala que en la UPA:

- El 78 por 100 ( $\mathrm{n}=61$ ) ha expuesto que no es una ley que sirve para que las mujeres reciban beneficios,

- El 22 por 100 ( $\mathrm{n}=17)$ dijo que sí.

En la UPB el 100 por 100 ( $n=9)$ han manifestado que no es una ley para que se beneficien las mujeres. 
«Herramienta eficaz en la labor policial»

El gráfico 7 indica que en las dos unidades policiales agregadas:

- El 85 por 100 ( $\mathrm{n}=74)$ de la muestra de guardias civiles aseveró que esta ley es una herramienta muy eficaz en la labor policial.

- El 15 por 100 (n=13) dijo que no.

Sobre esta cuestión, en la UPA:

- El 83 por $100(\mathrm{n}=65)$ contestó afirmativamente.

- El 17 por $100(n=13)$ dijo que no.

En la UPB el 100 por 100 (n=9) de sus componentes contestó afirmativamente a esta pregunta.

\section{GRÁFICO 7}

Distribución de la muestra de los guardias civiles con los que opinan que la Ley 1/2004 es una berramienta eficaz para el desempeño del cometido encomendado a las FF.SS.

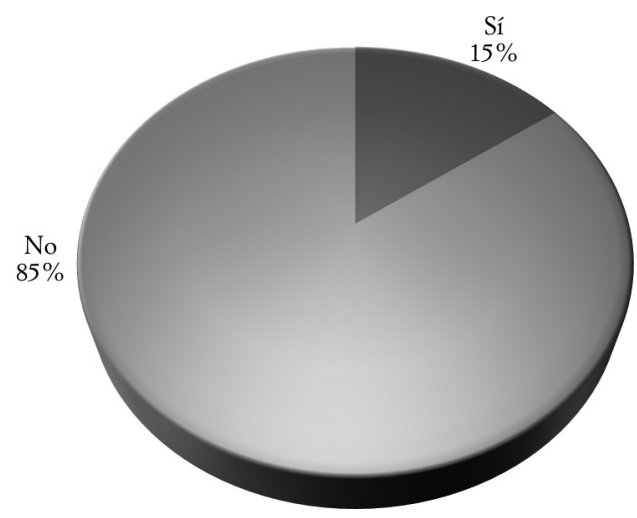

Fuente: elaboración propia, 2013.

Demandas. En esta ocasión se les preguntó qué medidas creen conveniente que serían necesarias arbitrar para el mejor desempeño del servicio en este campo, pudiendo contestar:

- Incremento del número de agentes dedicados a esta misión.

- Más formación en violencia de género.

- Otras que consideraran convenientes. 
«Incremento de agentes»

En el gráfico 8 se señala que en los datos agregados de las dos unidades policiales:

- El 47 por 100 ( $\mathrm{n}=41)$ de los guardias civiles interesaban el aumento de la plantilla con el objeto de dedicar más efectivos a la violencia de género.

- El 53 por $100(n=46)$ consideró que no era necesario.

En la UPA:

- El 46 por $100(n=36)$ interesa más agentes.

- El 54 por $100(\mathrm{n}=42)$ opina que no es preciso.

\section{GRÁFICO 8}

Distribución de la muestra de los guardias civiles con los que aluden la falta de personal en las dos UP agregadas

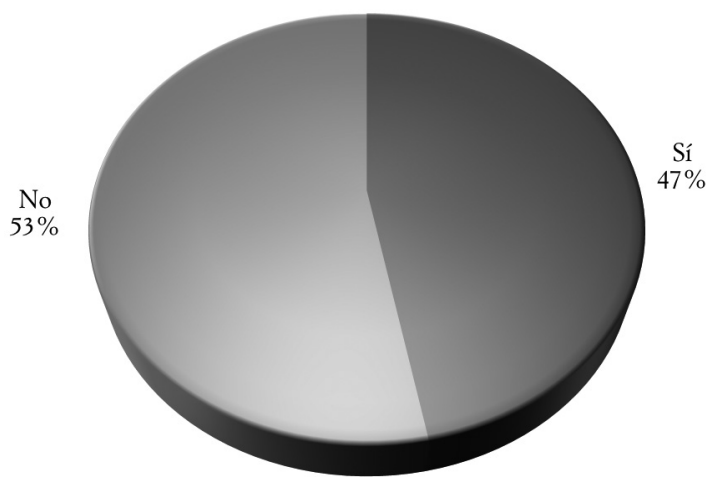

Fuente: elaboración propia, 2013.

En la UPB:

- El 56 por $100(n=5)$ han manifestado el deseo de incrementar el número de efectivos.

- El 44 por $100(n=4)$ dijo que no.

«Formación»

El gráfico 9 especifica en datos agregados de las dos unidades policiales: 
- El 54 por $100(n=47)$ del total de guardias civiles creen necesitar más formación en materia de violencia de género, porque consideran una temática muy delicada.

- El 46 por $100(n=40)$ opina que no es preciso porque tienen la preparación necesaria.

En la UPA:

- El 56 por $100(\mathrm{n}=44)$ demanda más formación.

- El 44 por 100 (n=34) dice que no.

En la UPB:

- El 33 por 100 (n=3) sí desearía más formación.

- El 67 por 100 ( $\mathrm{n}=6)$ piensa que no la necesitan.

\section{GRÁFICO 9}

Distribución de la muestra de los guardias civiles con los que desean adquirir más formación específica en esta materia, agregadas las dos UP

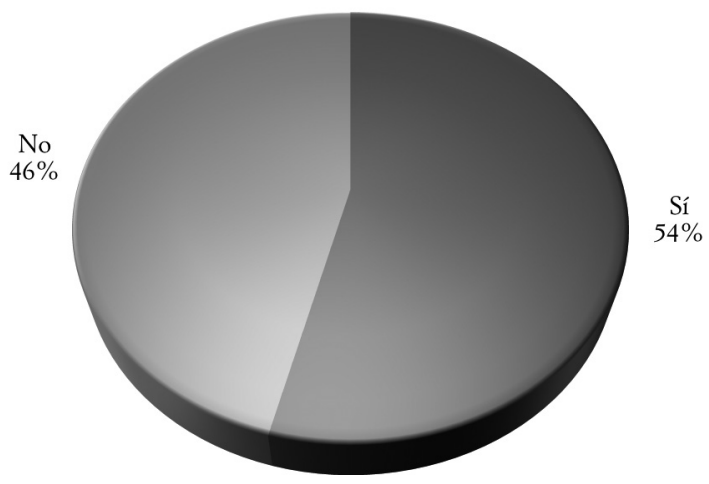

Fuente: elaboración propia, 2013.

Los que desearían una intervención más próxima de otras instituciones sociales representan el 16 por $100(n=14)$ del total de la muestra agregada, frente a los que dicen que no, un 84 por $100(n=73)$.

Como posteriormente se ha podido constatar en las entrevistas a los guardias especialistas en violencia de género, la colaboración con los demás servicios de carácter asistencial es óptima y actúan prontamente con eficacia 
cuando se les requiere para ello. Los que han demandado mayor intervención de estos servicios es muy probable que desconozcan esta circunstancia, puesto que las Áreas de Atención a la Mujer, Servicios Sociales Municipales y otras entidades similares contactan directamente, para asuntos relacionados con las mujeres víctimas, con los especialistas en violencia de género de los puestos principales o con el responsable de este ámbito en los puestos ordinarios, por tanto, la mayoría de los efectivos que componen las UP desconocen, porque no les compete, la labor de coordinación que se lleva a cabo entre los distintos servicios de atención a las mujeres víctimas.

3. En la hipótesis de partida se aseveraba que el cuerpo de la Guardia Civil presta un servicio de calidad en el ámbito de la violencia de género en las relaciones de pareja o expareja, y que esta labor de asistencia y protección a las mujeres víctimas se realiza de forma más eficiente en las unidades policiales con especialistas específicos en violencia de género, en contraposición a las unidades del instituto que carecen de ellos.

Para confirmar esta afirmación provisional, rechazarla o rectificarla, se ha diseñado un objetivo: analizar el grado de sensibilidad y profesionalidad que tienen los guardias civiles en el ámbito de la violencia de género.

Con respecto a los guardias civiles se ha podido constatar el grado elevado de sensibilidad que tienen con respecto a la violencia contra las mujeres: el 84 por 100 opina que es una lacra social que vulnera los derechos humanos de las mujeres. En la UPB este porcentaje asciende al 100 por 100 de la plantilla.

El 94 por 100 piensa que la violencia contra las mujeres en las relaciones de pareja o expareja no es un invento de las mujeres para obtener poder. En la UPB este porcentaje llega al 100 por 100.

Un 87 por 100 de los agentes contestó que la violencia de género no es exagerada, que es un problema grave. En la UPB el 100 por 100 de los guardias contestó que no es exagerada.

De nuevo se observa que la UPB obtiene unos resultados de mejor valor que la UPA.

Se ha podido constatar que los agentes que han contestado a estas preguntas negativamente, es decir, no consideran la violencia de género como una lacra, creen que es mal utilizado por las mujeres y exagerado, tienen poco o casi nulo contacto con las mujeres víctimas de violencia de género en las relaciones de pareja, eso indica el menor grado de sensibilidad, porque sí se ha podido apreciar que en los agentes que asiduamente intervienen con esta problemática, el grado de comprensión es diametralmente opuesto a los anteriores. 
Otra cuestión importante ha sido la relacionada con la LO 1/2004 de Medidas de Protección Integral contra la Violencia de Género. La gran mayoría de los agentes encuestados han indicado que es una buena herramienta para las fuerzas de seguridad, porque con la normativa anterior, al ser la mayoría de los ilícitos penales en este ámbito considerados faltas, no se podía dar una respuesta de seguridad y protección a la víctima.

En el ámbito de las demandas, dos son las solicitudes que han aflorado de manera patente: la falta de personal y la falta de formación. En relación con la primera, en las dos unidades policiales los agentes han puesto de manifiesto que se deberían ampliar las plantillas para dedicar más guardias de manera exclusiva y especializada a este campo de la violencia de género. En el caso de la UPB, exponen expresamente que personal especializado debería hacerse cargo, a nivel comarcal, de los casos de violencia de género en las relaciones de pareja o expareja, es decir, que varias unidades policiales pequeñas fueran asignadas a una unidad policial superior para investigar estos hechos delictivos específicamente.

En el caso de la formación, se necesita la preparación en sensibilización, porque adiestramiento en la implementación de los protocolos de actuación, instrucción de diligencias y atención a las víctimas, dan sobradamente cuenta de su óptima preparación, y así lo han declarado las mujeres víctimas en este estudio. También es posible que una gran mayoría soliciten más formación no tanto por las carencias que posean de ésta, sino por el grado de exigencia que los guardias civiles tienen por querer prestar un servicio de mejor calidad a estas mujeres.

Para finalizar, decir que la hipótesis de partida se ha cumplido parcialmente. La Guardia Civil realiza una labor de asistencia social y policial de calidad a las mujeres víctimas de violencia de género en las relaciones de pareja o expareja, pero no se hace de forma mejor en las poblaciones que poseen especialistas específicos en esta materia; al contrario, en las poblaciones pequeñas con unidades policiales de pocos agentes, el servicio es un poco mejor valorado por las mujeres víctimas, debido a la cercanía que en estas localidades existe entre la Guardia Civil y la ciudadanía a la que sirve. En las poblaciones de mayor número de habitantes, esta carencia de proximidad es suplida por la constante atención que dispensan los especialistas en violencia de género de estas unidades policiales, como así lo han expresado las mujeres víctimas. 


\section{BIBLIOGRAFÍA}

Centro de Investigaciones Sociológicas, Barómetros de los meses de febrero y de mayo [CIS, 2011 a) y b)]. http://www.cis.es/cis/opencm/ES/11_barometros/ depositados.jsp (consulta: 1 de mayo de 2013).

- Barómetro del mes de abril (CIS, 2013). http://www.cis.es/cis/opencm/ES/11_ barometros/index.jsp (consulta: 1 de mayo de 2013).

DiReCCIÓN GENERAL DE LA GUARDIA CIVIL, Información Institucional (DGGC, 2013). bttp://www.guardiacivil.es/es/institucional/estructuraorganizacion/orgperiferical index. html (consulta: 3 de septiembre de 2013).

Ley Orgánica 1/2004, de 28 de diciembre, de Medidas de Protección Integral contra la Violencia de Género (LO 1/2004), BOE, núm. 313, de 29 de diciembre de 2004.

Rodríguez, D., y Valldeoriolade, J., Metodología de la investigación, Barcelona, Eureca Media, 2009. 